\title{
Exploring Personalized Vibrotactile and Thermal Patterns for Affect Regulation
}

\author{
MUHAMMAD UMAIR, Lancaster University, United Kingdom
}

CORINA SAS, Lancaster University, United Kingdom

NIAZ CHALABIANLOO, Boğaziçi University, Turkey

CEM ERSOY, Boğaziçi University, Turkey

\begin{abstract}
The growing HCI interest in wellbeing has led to the emerging area of haptics for affect regulation. In such technologies, distinct haptic patterns are usually designed by researchers; however, current work provides a limited reflection on the rationale for the implemented patterns or the choice of haptic modality. We also know little about how people may benefit from engagement in designing such patterns and what design principles underpin them. We explored vibrotactile and thermal modalities to address these gaps and report on a study with 23 participants. These created haptic patterns for affect regulation during stress elicitation. Findings indicate that subjective and objective measures of anxiety and stress were lower in participants who received haptic patterns than those who did not, and highlighted key experiential qualities of vibrotactile and thermal patterns, and their potential for affect regulation. These open up new design opportunities for affect regulation technologies, including supporting implicit affect regulation through entrainment of slow bodily rhythms, decoupling it from predominant vibrotactile modality, designing thermal biofeedback patterns, and supporting personalized and adaptive patterns.
\end{abstract}

CCS Concepts: • Human-centered computing $\rightarrow$ User studies; Haptic devices.

Additional Key Words and Phrases: affect regulation, haptic patterns, vibrotactile, temperature, personalization

ACM Reference Format:

Muhammad Umair, Corina Sas, Niaz Chalabianloo, and Cem Ersoy. 2021. Exploring Personalized Vibrotactile and Thermal Patterns for Affect Regulation. In Designing Interactive Systems Conference 2021 (DIS '21), fune 28-fuly 2, 2021, Virtual Event, USA. ACM, New York, NY, USA, 24 pages. https://doi.org/10.1145/3461778.3462042

\section{Introduction}

Emotional wellbeing and affective health rely on people's abilities to become aware, understand, and regulate their emotional responses. A growing body of HCI work has focused on affective technologies more broadly, and in particular, those integrating biosensors [78]. Most of these systems tend to target emotional awareness involving predominantly visual interfaces for mapping physiological arousal captured through biosensors [55, 77, 87]. However, there is a growing body of work targeting affect regulation by using physical materials and actuators [8,51,98] such as haptics $[3,9,10,58,68,98]$. Affect (emotion, mood, stress) regulation refers to managing or adaptively responding to emotional experiences [30]. Being able to effectively diffuse high arousal negative affect can improve affective health and wellbeing, whereas the inability to moderate one's emotional responses can have detrimental impact. Technologies supporting affect regulation provide interventions that help users manipulate high arousal negative affect such as stress, anxiety, fear, or anger to help themselves calm down.

Permission to make digital or hard copies of all or part of this work for personal or classroom use is granted without fee provided that copies are not made or distributed for profit or commercial advantage and that copies bear this notice and the full citation on the first page. Copyrights for components of this work owned by others than the author(s) must be honored. Abstracting with credit is permitted. To copy otherwise, or republish, to post on servers or to redistribute to lists, requires prior specific permission and/or a fee. Request permissions from permissions@acm.org.

(c) 2021 Copyright held by the owner/author(s). Publication rights licensed to ACM.

Manuscript submitted to ACM 
Haptics offer tactile sensations that are embodied, subtle, private and easy to engage with, or disengage from [68, 98]. Previous research on the use of haptics for affect regulation [58] has suggested that haptics can act as distraction, cue specific thought patterns, or influence the experiential, behavioral, or physiological components of an emotional response all of which are important parts of regulation processes [31]. Haptic technologies targeting affect regulation involve vibrations aligned with slow heart rate $[3,9]$ or slow breathing pace $[59,68]$ whose target values can be provided without the need of biosensors for real-time measurement of these signals. In these cases, the haptic affective interfaces are intended to train users to lower their heart rate or breathing pace in order to regulate their physiological arousal. Besides vibrations, research on other modalities, such as thermal one for supporting affect regulation, has been limited. Prior work on the experiential and material qualities of thermal modality $[38,89,98]$ has suggested that, in comparison to vibrations, heat supports inward-looking sensory experience. Heat also feels comfortable, directing attention in a subtle and slow manner. These aspects suggest that heat may be a strong alternative modality for affect regulation.

While both affective technologies, supporting either emotional awareness or regulation, rely on increasing one's awareness and control of physiological responses, the mapping of such responses onto the interface elements such as colors or vibration patterns is usually provided by researchers and guided by design principles such as familiarity, ambiguity, and balance [87]. The value of users' engagement in the design of these mappings has just started to be explored for affective interfaces targeting emotional awareness [97]. However, we know little of how people may benefit from engagement in the design of vibration- or temperature-based tactile patterns for affective interfaces targeting regulation, and what design principles may underpin them. To address this, it is important to understand users' engagement with technology and its effectiveness for affect regulation before it is used in everyday life settings. In this paper, we explored vibrotactile and thermal stimuli for affect regulation, by engaging users in the exploration and creation of vibration- and temperature-based non-biofeedback haptic patterns. We also evaluated the impact of these haptic patterns on participants' affect regulation, following a standard stress elicitation method. In particular, we aim to answer the following research questions:

- What are the personalized vibrotactile and thermal patterns for affect regulation?

- How are they designed: which qualities and design principles underpin them?

- What is the value of these haptic patterns for affect regulation?

We report an exploratory study with 23 participants who were assigned to one of two groups. One group created either vibrotactile or thermal patterns for affect regulation and subsequently received these personalized patterns through the use of haptic actuators during a stressor task. The other group took part only in the stressor task without any such haptic patterns. To understand the rationale behind personalized haptic patterns and their effectiveness, we conducted semi-structured interviews with those participants who received haptic patterns. We also collected self-reported measures of anxiety as well as physiological heart rate data related to stress from all participants. We found that participants in both haptic groups, i.e., vibration and thermal patterns, had a lower subjective level of anxiety assessed through the State-Trait Anxiety Inventory (STAI) than participants who received no haptic patterns. At the same time, the heart rate variability (HRV) feature of those participants who received haptic patterns also changed, indicating lower stress, especially under low frequency vibrations, albeit not significantly. Moreover, STAI scores suggest potentially stronger beneficial impact on subjective experience of anxiety of cool temperatures and low frequency vibrations.

The main contributions of this work include (i) fresh insights into the experiential qualities of vibrotactile and thermal patterns for affect regulation, (ii) empirical evidences for personalized haptic patterns' impact on subjective 
and objective anxiety and stress regulated during stressor tasks, and (iii) design implications for affect regulation technologies highlighting the value of implicit affect regulation through entrainment of slow bodily rhythms, of decoupling such entrainment from the predominant vibrotactile modality, of thermal biofeedback leveraging thermal patterns, and of personalized and adaptive patterns.

\section{Background}

\subsection{Affective Interfaces in $\mathrm{HCl}$}

Over the last decade, much research in HCI has focused on affective interfaces. Whether they integrate biosensors or not, most of such technologies aim to support emotional wellbeing through increased relaxation, self-awareness, reflection, or affect regulation $[5,78]$. Systems that integrate biosensors support the important function of providing historic or real-time biosensory feedback on users' physiological states measured in terms of arousal or valence. While relaxation or mindfulness have also been supported through neurofeedback [11,79], common biosensors capturing aspects of the autonomous nervous system include heart rate variability (HRV) or galvanic skin response (GSR) whose feedback is often provided in visual [77, 96, 97] or haptic modalities [9, 98].

Visual representations often employ shapes' sizes and colors on desktop [55], mobile phone screens [77], or smart materials [96] to represent emotional arousal. Affective diary [87] is an early system integrating GSR and pulse sensor, as well as pedometer to map bodily experiences onto abstract, anthropomorphic silhouettes whose color conveyed arousal, and whose shape conveyed the amount of movement. The design of these mappings was informed by previously suggested design guidelines of balancing familiarity and ambiguity in abstract representations of emotions in order to support users' understanding of them. These visual mappings are aimed to support users' affective interaction or sense-making of their emotional experiences rather than prescribe interpretations. While Affective diary provided historic visualizations, Affective health [77], a follow-up system from the same research group, integrates GSR sensor and accelerometer for real-time visualizations of arousal in a spiral-shaped form using color red and blue for high and low arousal, respectively, and spiral width to communicate amount of movement. Another example is AffectAura that integrates GSR sensor and uses the size of bubble shapes to represent the intensity of arousal, and their color to represent emotional valence on a desktop interface with the aim to support emotional reflection over longer periods of time [55]. Smart materials-based affective interfaces have also been designed, integrating GSR sensors to provide real-time visual feedback on changes of arousal through thermochromic paints [95, 98]. In contrast to visual, haptic modality has been less explored. The limited efforts in this direction have focused for instance on mapping changes of arousal captured through GSR sensors to shape-changing materials through vibration, squeezing, or heating wristbands [98].

Despite the growing interest in designing biofeedback-based affective interactions, a remaining challenge is the mapping of biodata to interface modality and its components. Much of the work in this space, including the systems highlighted above, have usually mapped color red, or larger shape sizes to high arousal, and color blue, or smaller shape sizes to low arousal. However, making sense of one's biodata and mapping it to representations and modalities that are easily recognizable and understood is not trivial. For instance, recent work proposed the soma design approach for exploring the qualities of biodata by materializing it through different sound and haptic modalities [1]. This ensures that through the first-person experience, biodata can be viscerally felt and understood by designers so that it can become meaningful somadata to more robustly inspire and inform design. While strong contributions to the field, such approaches align with most HCI work on affective interfaces describing research-driven mappings from bodily data to 
interface elements. In contrast, users' engagement in the design of such mappings has been limitedly explored. A few exceptions include new design methods for people to codesign personalized flavors for emotional communication or regulation [24, 25], or toolkits for end-users to assemble their own personalized affective interfaces targeting anxiety [84], or emotional awareness [97]. Key findings from these approaches include strong benefits from involving users in the design process such as users' increased agency and control, improved understanding of the inner workings of technology, and more importantly, the creation of personalized interfaces for their specific needs. However, such work has seldom focused on users' generation of personalized mappings with only a few exceptions here have looked mostly at visual modality in order to map changes of arousal to visual interfaces [97].

To summarize, most of the previous research on affective interfaces has built on the initial design principles of familiarity, ambiguity and balance [87] in order to provoke, entice, and empower users to better understand their bodily experiences and emotions, as these are central to how people interpret and make sense of the world. However, it is surprising that the mappings between bodily data and the interface elements have been limitedly designed by the users, given that such personalization may account for stronger engagement and user experience. In addition, in this space, the visual modality has received significant attention for emotional awareness or regulation, than the haptic one for affect regulation $[55,77,87]$. Thus, we now turn our attention to haptic based interfaces and particularly those for affect regulation.

\subsection{Haptics for Affect Regulation}

Affect regulation is referred to as managing and adaptively responding to emotional experiences [8, 30]. Being able to effectively diffuse negative emotions can improve affective health and wellbeing, whereas the inability to moderate one's emotional responses has negative impact. People regulate to increase pleasant emotions (happiness or joy) or decrease unpleasant ones (fear, anger, sadness). Alongside the more mature research area focused on designing for affective awareness and reflection in daily lives, there has been also a growing interest in haptic interfaces $[3,9,10,51,58,68,98]$ to regulate high arousal negative affect. Such technologies tend to provide real-time interventions that help users manipulate their affective states in order to calm down under pressure. The choice of haptics is not surprising, as haptic actuators can carry emotional information [75]. They offer tactile sensations that are subtle, private, and embodied and have been recently explored both in the research and commercial technologies for real-time affect regulation $[3,9]$.

Affect can be regulated at each of the five phases namely situation selection (taking actions that make it more or less likely that one will be in a situation entailing desirable or undesirable emotions), situation modification (taking actions that directly alter a situation changing its impact), attention deployment (directing one's attention to influence affective response), cognitive change (modifying one's appraisal of a situation to alter its impact) and response modulation (directly influencing experiential, behavioral, or physiological components of the affective response after it is well developed) [30, 31]. Previous research on haptics and affect regulation has suggested that haptics can act as distractions, cue specific thought patterns, or influence the experiential, behavioral, and physiological components of affective response by simulating bodily response for low emotional arousal [58]. Examples of haptics for response modulation are vibrations delivered at 60 beats per minute (bpm) without the aid of real-time biosensors in order to guide users to down regulate their arousal during stressor tasks [3, 9].Previous findings also indicate that people use perceptual properties of vibrations, emotional interpretations, and metaphors to assign familiar meanings to vibrations [82]. Indeed, studies exploring vibrotactile patterns for regulation have found that vibrations delivered at a frequency $30 \%$ lower than the baseline heart rate can help decrease anxiety and increase heart rate variability during a stressful event, while fast 
patterns at a frequency $30 \%$ higher than the baseline heart rate can lead to an increase in self-reported anxiety and heart rate variability [10].

With a few exceptions, other haptic modalities for affect regulation such as thermal one have been less explored. A noticeable exception is Soma Mat [89] which provides heat stimuli to shoulders, hips, and calves to support attention to one's body during Feldenkrais exercises. Through an experiential exploration of heat as a material for design [38] and a subsequent user study, authors highlighted the subjective nature of thermal stimuli marked by individual differences in sensitivity to heat and appreciation of it, as well as its subtleness when provided within a comfortable range. Authors also indicated that unlike other haptic modalities such as vibration or touch which are typically associated with external stimuli, heat has the potential to be perceived from inside the body.

Work exploring both vibrotactile and thermal patterns for emotion awareness and regulation is yet rare. For instance, Umair and colleagues [98] employed smart materials to explore a range of prototypes including vibrotactile motors and heating elements actuated by changes in physiological arousal measured through GSR sensors. Their findings highlight temporal qualities with heat actuators being less responsive and more inertious when compared to vibrations, as the former need more time to respond and deactuate because of the accumulated heat. As a material for design, heat has the potential to grab attention and it is also perceived as subtle and comfortable $[38,98]$.

Within this emerging HCI interest in haptics for affect regulation, the focus has been mostly on vibrotactile actuation for guiding users towards slowing down their autonomous nervous system response. In contrast, thermal patterns for affect regulation has been less explored, albeit its subtle and comfortable qualities make it a strong candidate modality. Our focus on the thermal modality for affect regulation is because of its experiential and material qualities i.e. comfortable, subtle, can direct attention, slow, and private. However, given the larger body of work on vibrotactile patterns for affect regulation, we decided to extend our exploration of thermal patterns merely in itself, to include also its comparison with vibrotactile patterns. Another direction in which our work extends the state-of-the-art is exploring the design of personalized haptic patterns for affect regulation by engaging users in their design. We involved users in the exploration and creation of vibration- and temperature-based non-biofeedback haptic patterns (in other words the patterns are not triggered by biosensors' data). We report on an exploratory study to investigate these patterns and their value for affect regulation.

\section{Method}

The aim of this work is to engage users in the design of personalized haptic patterns for affect regulation, and to study the impact of these patterns on participants' affect regulation during a standard stress elicitation task. The overall study was facilitated by the first two co-authors and consisted of individual sessions, each lasting about 60-70 minutes. The study took place in a soundproof room with no auditory or visual distractions and a consistent physical layout: participants sat on a chair facing a small table towards the wall. We now describe in detail participants' recruitment, study procedure, data gathering and analysis. Participants were recruited through mailing lists and advertisements in the university campus, with eligible criteria requiring that they had no history of depression, anxiety, or cardiovascular disease. The sample consisted of 23 participants, 11 male, 12 female, average age 25.4 years, (range 20-38 years), which was randomly split in haptic group exposed to either vibrotactile patterns ( 7 participants) or thermal patterns (8 participants), and no haptic group (8 participants).

Participants in the haptic group first created their personalized patterns for affect regulation, either in vibrotactile or thermal modality, and subsequently used respective actuators in order to receive their personalized patterns throughout 


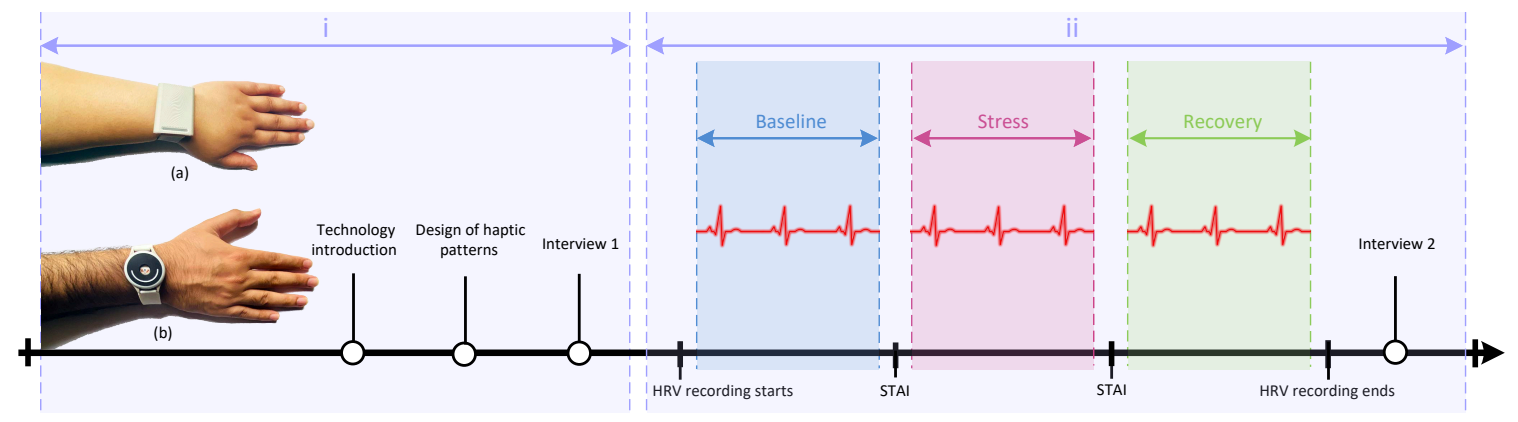

Fig. 1. Study procedure: i) users personalizing haptic patterns (haptic group) ii) stress elicitation task (haptic and no haptic group)

the stress elicitation task. The no haptic group took part only in the stress elicitation task without any haptic patterns and did not personalize any haptic patterns (Figure 1).

\subsection{Users' Design of Personalized Haptic Patterns for Affect Regulation}

The aim of this part was to engage users with haptic actuators to explore and personalize their haptic patterns for affect regulation. This study part consisted of 3 stages further described. In the technology introduction stage we introduced the haptic technology consisting of two commercial, wrist-worn devices for actuating temperature (Figure 1a) [46], and vibration (Figure 1b) [14], respectively. Both actuators have been previously used in HCI research to deliver vibration [3] and temperature-based haptic patterns [85]. Participants were given verbal and visual, slide-based descriptions of the actuators and how to use them. This stage lasted 10 minutes and was attended only by participants in the haptic group, after which they were randomly allocated to the two subgroups, so that each participant experimented with only one type of haptic actuator: either vibration (7 participants) or temperature (8 participants). This decision was taken in order to limit participants' exposure to the stress inducing task, prevent fatigue, and keep the study duration roughly within 1 hour.

After the introduction, participants in the haptic group took part in the main stage, namely the design of haptic patterns for affect regulation. For this, we instructed them to engage in the exploration of the provided actuators and create vibrotactile- or temperature-based patterns on the wrist, in order to calm themselves down when feeling stressed. Each haptic actuator was connected to a mobile application via Bluetooth, through which participants could explore and change the actuators' parameters. For both, participants could place the actuators on either inner or outer side of the wrist. Vibrotactile actuator provided vibrations at a specific frequency. For simplicity, we will refer to vibration frequency as beats per minute (bpm). The actuator enabled participants to vary its frequency (from 30 to $185 \mathrm{bpm}$ ) as well as vibration intensity ( $5 \%$ to $100 \%$ - vibration force at $100 \%$ intensity: $1.10 \mathrm{G}$ ) [16]. The vibration motor produces an acceleration rate of $0.83 \mathrm{G}\left(8.14 \mathrm{~m} / \mathrm{sec}^{2}\right)$ at $76 \%$ intensity and $0.05 \mathrm{G}\left(0.49 \mathrm{~m} / \mathrm{sec}^{2}\right)$ at $5 \%$ intensity. The motor sat in a metal base and a plastic cover. The overall unit was $38 \mathrm{~mm}$ in diameter, $9 \mathrm{~mm}$ thick, and weighed 20 grams, being held together by a silicon strap (10 grams). Thermal actuator enabled participants to vary, through its purpose built app, the temperature intensity within the range of $-11^{\circ} \mathrm{C}$ to $+16^{\circ} \mathrm{C}$ from user's baseline temperature. This allowed them to create thermal patterns representing either increase up to $+16^{\circ} \mathrm{C}$, or decrease down to $-11^{\circ} \mathrm{C}$. In order to prevent thermal accumulation and to maintain a specific temperature, the actuator provided thermal patterns in intervals of about 7 seconds, followed by a gap of 7 seconds mitigating the accumulation of heat over time. The personalization 
of vibrotactile and thermal patterns was to an extent limited by the constraints of the two devices we chose to work with, that allow varying the vibration frequency and intensity, and temperature of thermal patterns only within the ranges specified above. We consider these parameters as suitable starting point of this exploratory study without being overwhelming, while future work building on our outcomes can look at a broader set of parameters including for instance frequency of actuating the thermal patterns, or adaptive change of frequency throughout the session based on users' stress level.

This stage lasted for 10 minutes, and was followed by brief individual semi-structured interviews (interview 1) where we asked participants to describe their bespoke haptic patterns, how, and why they believed that such patterns could help regulate stress.

\subsection{Impact of Personalized Haptic Patterns on Affect Regulation during Elicited Stress Task}

Once the design of haptic patterns was completed, we aimed to assess their impact on affect regulation following a standard stress elicitation procedure consisting of baseline, stressor task, and follow up recovery [45]. Participants in the haptic group received the vibrotactile or thermal patterns that they designed in the first part of the study, through their respective actuators, while participants in the no haptic group received neither actuators nor patterns. This procedure consisted of three stages.

The first stage was the baseline measurement of stress level, during which, participants sat in a comfortable posture for 10 minutes with their hands on the table while avoiding any large movements.

After this stage ended, we started the stressor task by replicating standard stress induction tasks successfully used in a number of studies [44, 81, 90]. Participants were exposed to 10 minutes of induced stress, and in order to avoid habituation and fatigue, we used two separate stressors, for 5 minutes each, delivered on a tablet (Samsung Galaxy Tab A 10.5"). One of the stressor was the Stroop Color and Word Test (SCWT), a neuropsychological test commonly used as a stressor in both clinical and non-clinical studies [81,90]. It requires participants to name a color on the display, shown together with a word naming colors, that either matches or does not match the shown color. The task leads to longer response time and larger number of errors when the color and words are not matched. The other stressor consisted of mental arithmetic tasks which is a component of Trier Social Stress Test (TSST) [44], a much used naturalistic psychological stress protocol [103]. Participants were instructed to count backwards for 5 minutes, with different instructions (e.g., counting backwards in steps of 13 from 1700), and say their answers out loud while one of the researchers pretended to take notes of the right and wrong answers.

Once the stressor tasks ended, haptic actuators were stopped and a 5 minute rest period was ensured to allow for recovery. This was followed by final individual semi-structured interviews (interview 2) with participants in the haptic conditions about their subjective experiences of the personalized haptic patterns and its perceived impact on the down regulation of stress. We also asked if they would revise the design of their haptic patterns, and their view on vibrotactile or thermal patterns for the regulation of high-arousal negative affect in daily life. These interviews lasted about 15 minutes. Both first and final interviews were audio recorded and fully transcribed for data analysis using a hybrid coding approach [20].

Besides interviews, we also recorded subjective and objective measurements of anxiety and stress. Stress is associated with feelings of mental discomfort, concerns, strain, and even anxiety. It often co-occurs with a condition that induces these feelings, such as a challenging quiz, examination or recruiter's interview [63]. To obtain the subjective measurements of anxiety, we used the State-Trait Anxiety Inventory (STAI) [86]. All participants were asked to fill out the paper-and-pencil versions of STAI questionnaire right after baseline and stressor task [64] as shown in Figure 1. 


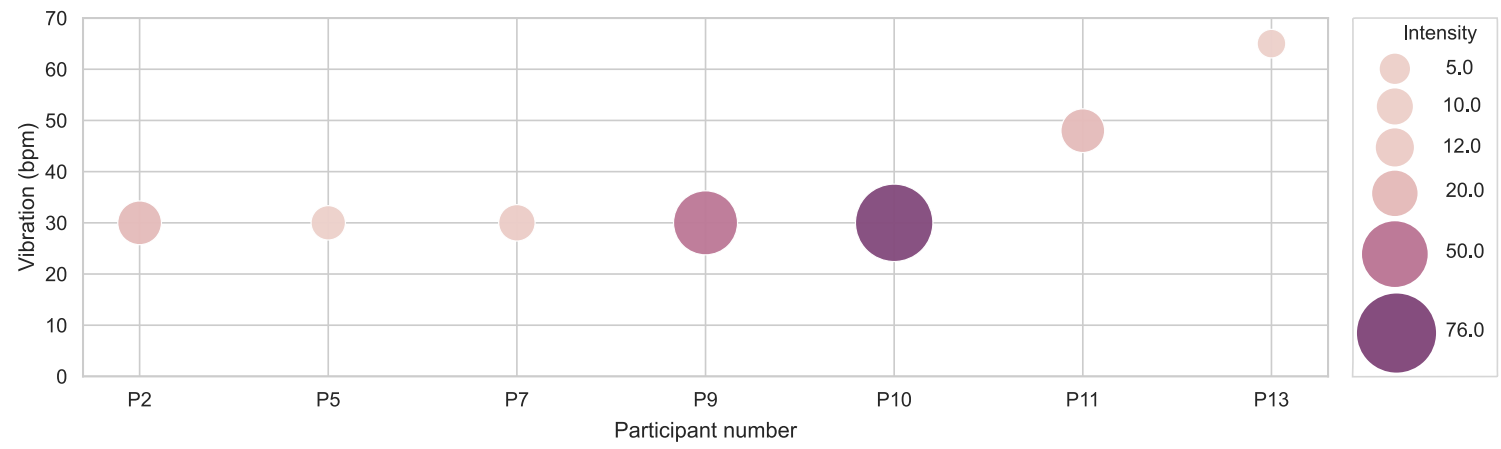

Fig. 2. Personalized beats per minute (bpm) and intensities of vibrations created by each participant.

The State-Trait Anxiety Inventory (STAI) questionnaire [86] comes in two forms, capturing self-reports of state or trait anxiety [48, 52]. We used STAI-Y-1 questionnaire consisting of 20 items (e.g. "I feel upset", "I feel secure") to measure state anxiety, defined as momentary emotional response resulting from situational stress such as examination [39], challenging cognitive tasks and particularly stressor tasks like SCWT [56], and TSST [93]. Each response was rated on a 4-point Likert scale, with high STAI-Y-1 scores indicating higher levels of anxiety. For simplicity, this scale is referred to in the paper as STAI.

For objective measurement of stress, we utilized heart rate variability (HRV), a commonly used physiological response for affect regulation [26, 43, 54]. HRV reflects autonomic balance, and represents changes in the time interval between successive heartbeats, also called inter-beat intervals (IBIs). A range of metrics have been developed based on HRV data. We chose to employ one of the most common time-domain features, i.e., root mean square of successive differences of the RR intervals (RMSSD). RMSSD is closely associated with parasympathetic activity [83], with findings confirming that decrease in RMSSD is a marker of increased stress, while increase in RMSSD indicates rest and recovery [91, 107]. Each participant wore a Polar H10 sensor which recorded time synced HRV data [28]. Polar H10 is a chest band which records HRV of a quality comparable to medical-grade heart rate monitors [28, 94]. Participants were told that the device measures physiological signals, but not which specific ones, to avoid any bias in the perception of the haptic feedback, i.e., the association of vibration to heartbeats.

\section{Findings}

We now describe participants' personalized vibrotactile and thermal patterns and their design, the perceived and actual value of such personalized haptic patterns for down regulation of stress, and their views regarding the use of haptic technologies for affect regulation in daily life.

\subsection{Personalized Haptic patterns for Affect Regulation}

In this section, we present the created vibrations, and thermal patterns, their experiential qualities, and participants' felt experience with them.

4.1.1 Vibrations on the Wrist. Most participants started to explore and personalize the vibration's frequency by varying the beats per minute (bpm) between the available range supported by the actuator, between the highest (185 bpm) and 
the lowest (30 bpm) values. Most participants (P2, P5, P7, P9, and P10) created vibrations with frequency of $30 \mathrm{bpm}$, as shown in Figure 2, while the others two chose higher frequencies (48 bpm - P11; 65 bpm - P13).

Interestingly, four out of seven participants associated the vibrations with their heart rate. They created a lower vibration frequency that would represent a slow heart rate $(30 \mathrm{bpm})$, or an ideal heart rate $(65 \mathrm{bpm})$ during high arousal negative affect. Previous research $[3,10]$ on the use of vibrations for emotion regulation has suggested that low frequency vibrations between $40 \mathrm{bpm}$ and $65 \mathrm{bpm}$ can make people less anxious, however, participants in our study chose an even lower frequency value (30 bpm - P2, P5, P9, P10), or the same upper value (65 bpm - P13). Interestingly $30 \mathrm{bpm}$ was the lowest vibration frequency that the actuator could support suggesting the value of even lower paced vibrations for affect regulation during stress.

Participants who chose $30 \mathrm{bpm}$ mentioned that higher frequency values felt "agitating" - P2, "strong" - P5, and "anxious" - P10. For instance, another participant mentioned that vibrations at higher frequency made him feel panicky whereas at $30 \mathrm{bpm}$ he felt like slowing down: "I went for the lowest [30 bpm] I could get. The reason is that I felt slower. It was nicer to calm down at a slow rate, rather than when it's really high [that] it's like more panicky" - P9. This participant also related these low frequency vibrations to an ideal pulse: "It is like a nice slow pulse. This is what your heart should $b e "$. Participant 2 who also used $30 \mathrm{bpm}$ mentioned that locking into their slow and fixed-tempo made him feel in tune with the beats: "I created a pattern that is very low in beats per minute. It is low bpm just because I'm thinking about a situation where you want to calm down because it seems slower than my heart rate and has a nice consistency. It gave me kind of a reference beat to focus on, which slows everything down for me to take my mind away, be able to control and regulate my breathing" - $\mathrm{P} 2$.

Similarly, P13 also associated the vibrations to their heartbeat. This participant created a $65 \mathrm{bpm}$ rhythm using her resting heart rate as a baseline. She mentioned that feeling a $65 \mathrm{bpm}$ heartbeat rhythm on the wrist would help her bring the increased heart rate down during intense fear: "I was considering the intense negative emotion of fear. In the state of fear, your heart rate increases. I chose 65 beats per minute that which is closer to the normal heart rate of a person and will help to calm down and normalize the heartbeat". An important finding is that these participants could relate the rhythm of the low frequency vibrations they created to the targeted heart rate, confirming previous findings [9]. Only two participants (P7 - 30bpm; P11- 48 bpm) did not associate the vibrations to heartbeat. Instead, they valued the slow rhythms created by vibrations: "I went for the lowest rhythm [30 bpm]. It feels slow and soft. The higher one was too much vibration for me" - P7. P11 also appreciated the slow rhythm of vibrations: "It is quite slow and kind of like rhythmic and not too intense. It calms you down with slowness" - P11.

Participants also manipulated the intensity of vibrations, and hence of their vibrotactile sensations. While frequency is linked to vibration's rhythm, intensity represents the strength of vibration. Findings indicate two main preferences, with most participants choosing intensities lower or equal to 20 percent, while the remaining two (P9 and P10) preferred it higher. The five participants (P2, P5, P7, P11, P13) who chose lower intensities reported that they did not want to experience strong vibrations when they try to calm down. Participant 5 describes the experience of feeling low intensity vibrations on the wrist as "soft" - P5. Similarly, P7 added: "When I started, I went to high intensity and I felt like it made me more nervous. So when I went down the scale, the gentle touch of the vibration made me calmer". This is an important outcome, indicating the value of directly experimenting with different levels in order to identify what feels right for each participant. Indeed, people perceived physical sensations differently, with previous research showing that different ways in which people perceive a stimulus depend upon an individual's learning, emotions and expectations [53]. Participant 9, for example, set the intensity to be 50 percent and P10 to be 76 percent. Both these participants mentioned that they did not want to create intense vibrations which would be distracting, yet not very low in intensity 


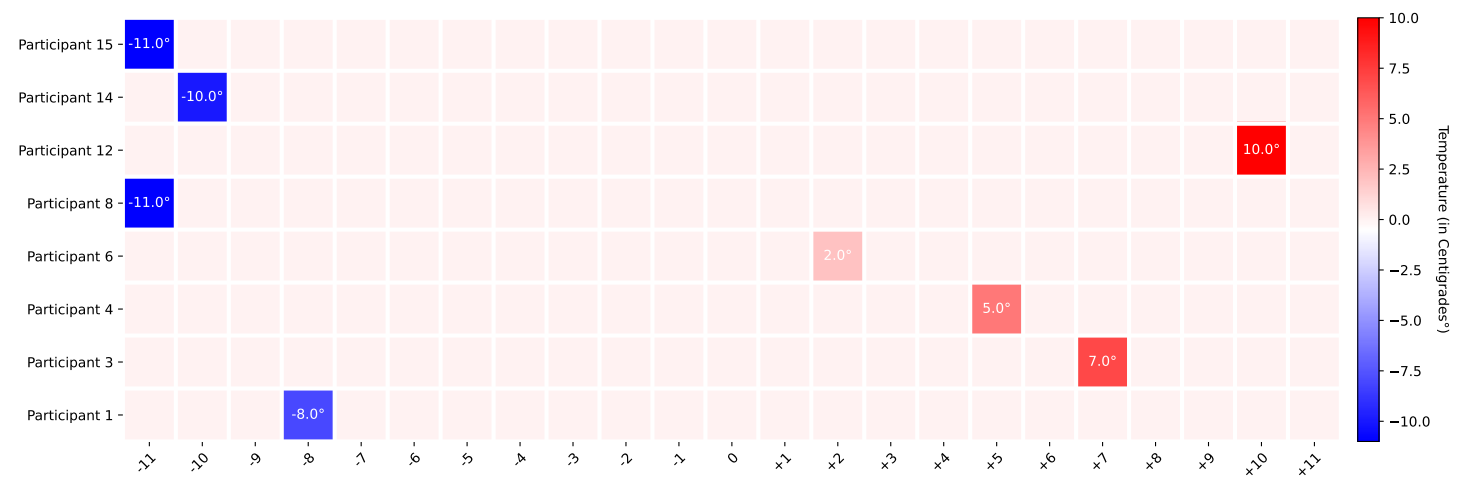

Fig. 3. Absolute temperature values (warm/cool) in ${ }^{\circ} \mathrm{C}$

either, since they could go unnoticed. Thus, the values they chose felt just right: "as for the intensity, you don't want it to be so intense that it's distracting. I chose 50 percent so that I could somewhat feel the vibrations. If it is something lower, you might just forget it" - P9.

4.1.2 Thermal patterns on the Wrist. For the design of the thermal patterns, participants started exploring wrist related locations. They experimented by placing the actuator on both the inner, and the outer side of the wrist, while actuating both high and low temperatures of the warm and the cool. In this way, they all discovered that the same temperature, be it high or low, was experienced differently based on the actuator's placement on the wrist. Most participants mentioned that they prefer temperatures at higher intensities on the outer side of the wrist, as the inner side is more sensitive to temperature changes. This shows different sensitivity for discriminating temperature on different parts of the wrist and the benefit of being able to adapt the temperature to account for it. Indeed, thermal sensitivity is not uniform across the body [106]. The number and density of thermoreceptors vary across the skin which creates a difference in conduction velocities of the warm and cool stimuli, leading to differences in perception [37]. The majority of participants (P3, P4, P8, P14, and P15) chose the inner side of the wrist for thermal patterns because they felt that it was more sensitive and they could more easily feel the temperature compared to the outer side of the wrist.

The temperature actuator could support temperatures between $-11^{\circ} \mathrm{C}$ to $16^{\circ} \mathrm{C}$ range, which participants placed on their skin, in standard room temperature settings $\left(21^{\circ} \mathrm{C}\right)$. Findings indicate two main preferences for either increase, or decrease of temperature from the baseline level. Half of participants (P3, P4, P6, and P12) chose thermal patterns by increasing the temperature by $7^{\circ} \mathrm{C}, 5^{\circ} \mathrm{C}, 2^{\circ} \mathrm{C}$, and $10^{\circ} \mathrm{C}$, while the other four participants $(\mathrm{P} 1, \mathrm{P} 8, \mathrm{P} 14, \mathrm{P} 15)$ chose to decrease the temperature by $-8^{\circ} \mathrm{C},-11^{\circ} \mathrm{C},-10^{\circ} \mathrm{C}$, and $-11^{\circ} \mathrm{C}$, respectively (Figure 3 ).

Interestingly, all participants choosing higher temperatures referred to heat as warmth and did not go beyond 10 degrees centigrade as it felt rather hot: "first I explored three to seven degrees, and after I tried a higher temperature, it felt too hot" - P3. All of those choosing higher temperatures mentioned that they found the feeling of warmth as comforting. Previous findings on experiential qualities of heat [38] have indicated that for most participants the experience of warmth was relaxing and comfortable. Participants in our study also related the experience of warmth on the wrist as comforting: "it is comforting because it gives me a sense of warmth. The feeling of warmth on my wrist is relaxing and makes me feel better" - P12. The remaining participants P6 and P4 related the feeling of warmth to human touch: "it 
feels like someone is holding my hand" - P6. P4 added: "When it's warm, it is kind of like associated with other human beings, touch, or hug or, kind of like emotional" - P4.

The other group of participants provided different reasons for choosing the thermal patterns with decreased temperatures. They reported that the feeling of cool on a particular place such as wrist is quite unique and unfamiliar as they are more used to getting or feeling the heat in everyday life rather than cool: "it is quite different. It's like putting the ice cubes on your wrist, which [...] kind of cools you down" - P8. Existing work has explored experiential qualities of heat; however, cool as a thermal modality has been under-explored [38, 98].

Interestingly, similar to previous group which preferred warmth rather than hot, participants in this group preferred cool rather than cold, as reflected in their rationale and language. Through experimentation, all these four participants discovered that cool feels more pleasant than warmth, so that they all chose values at $-8^{\circ} \mathrm{C}$ or lower. This preference for cool was usually expressed in contrast to the perceived negative experience of heat. They found cool as more pleasant since increasing heat made them feel "hot" - P1 and "nervous" - P15. P1 also pointed out that "compared to higher heat which felt intense, cool is just more relaxing and is naturally calming to me". These findings confirm existing research on thermal stimuli showing that heat risks being associated with higher arousal and perceived less pleasant compared to cool $[75,76]$. Both participants $\mathrm{P} 8$ and P15, who chose the lowest temperature $\left(-11^{\circ} \mathrm{C}\right)$ the actuator could support, mentioned that they would have used even lower temperatures if possible: "I chose $-11^{\circ} \mathrm{C}$ which is not freezing cold but quite a pleasant sensation. I think coolness is much more pleasant than the heat. Initially, I was working with heat but it was making me nervous so then I moved towards the cool which felt very pleasant" - P15.

All participants who chose lower temperatures mentioned that besides its pleasant nature they would like to feel cool because the body temperature rises during an intense negative situation. Thus, the actuator's contrasting thermal patterns on the body will help them notice and "cool off": "If I am in a heated situation where I'm all angry and heated up, this coolness will help me regulate" - P14.

\subsection{Participants' Experiences of Haptic Patterns for Affect Regulation}

4.2.1 Haptic Patterns during Stressor Task. All participants mentioned that they could feel vibrations or temperature on the wrist while concentrating during the stressor task. In both temperature and vibration conditions, when participants felt increasing stress, their focus shifted more towards the stressor task, however, consistent tactile sensations kept their presence known.

In vibrotactile group, the vibration frequency was consistent and grabbed attention from time to time: “it's physically there, it grabs your attention. My attention just kind of switched to it then without me, necessarily, doing it myself. Every now and again, it takes you away from what you're doing" - P5. Once participants engaged with the vibrotactile rhythm, they were able to follow it: "when I got into the rhythm, it definitely helped me calm down" - P11, while concentrating on the task: " $i$ was aware of these vibrations that I was getting while mentally concentrating on the questions" - P7. Similarly, other participant added that the rhythm acted as a reference during the stressor task: "I think when you feel yourself getting stressed out, you could just reference the vibrations, which were always there, and consistent. The vibrations did help just providing a reference point. I felt calm doing it [stressor task] and it felt slower and slower" - P2. P13 also pointed out that the vibration rhythm helped her relax during the stressor task: "when I got in a flow [rhythms] I was able to get hold of it. So, after that point I was able to relax".

Unlike vibrations, temperature changes are slow and more embodied. Research on thermal patterns shows that people are able to detect even one degree change in temperature [106]. When compared with vibrations for affective feedback, 
temperature changes were considered more expressive and noticeable [98]. During the stressor task, increasing warm and cool sensations made participants pay attention to them: "I think initially when I started the stress test, I scored very high and I was mentally very active. I totally forgot about the cool but then out of a sudden I felt cool. It felt pleasant and grabbed my attention [...] It kept on cooling me down. I think if I wasn't using such a device, I would have been more stressed" - P15. This is an illustrative quote indicating the perceived value of cool temperature patterns for down regulation during stress. For P8, the felt experience of cool was strong, which distracted him in a positive way, each time the temperature decreased: "the coolness just distracted me [...] I was absolutely calm in the sense that it didn't bother me that I can't keep up with those [stressor] requirements or be right or wrong".

4.2.2 Haptic Patterns after Stressor Task. Reflecting on the experience of tactile patterns during the stressor task, two thirds of participants reported that they would like vibration or temperature intensity to change during the stressor task. Participant 10 mentioned that she would prefer vibrotactile intensity to vary as the stress increases or decreases: "I think the intensity can be higher or lower. If it can be in proportion [...] if you are more stressed to be more intense, or the other way". As these participants engaged in the stressor task, their perception of tactile sensation changed and they wanted stronger intensity to keep their attention when they were more stressed. P12 noted that she would like to feel more heat on the wrist as the stress increased: "I would change the intensity of the temperature. I would want it to be a bit higher in order to feel it more". Similarly, P6 added: "when I tried the heat in the beginning, I wasn't as stressed but if I am in the middle of a stressful situation then I need more warm temperature". Two participants mentioned that they would prefer a decrease in the intensity towards the end of the second stressor because constant tactile sensations on the same place on the wrist felt a bit too much: "the first one [stressor] was absolutely fine; however in the second one, I would prefer maybe less intensity so that I can feel like it is [vibrotactile patterns] there but I cannot feel it as much" - P11. P3 also added that she would prefer less heat during the last part of the stressor: "I still think heat would work better than the cool but I would not want it to be hot. The last three minutes [towards the end] felt a little hot so I would want to bring it to a lower degree".

4.2.3 Haptics for Affect Regulation. In addition to the interviews, to further explore the impact of haptics on subjective and objective measures of anxiety and stress, we also employed a between-subject design. The choice of between-subject over within-subject design was informed by the need to minimize participants' exposure to the stressor task and limit the carryover effect [29]. Given that our data did not meet the assumptions of normality and homogeneity of variance, we used Kruskal-Wallis (K-W) test, a non parametric equivalent to one-way ANOVA [34, 62]. Kruskal-Wallis is an omnibus test which only detects that at least two groups among all groups are significantly different. Application of multiple pairwise K-Ws instead of a post hoc test increases type-I error, requiring error correction methods such as Bonferroni [33]. However, in our analysis we performed K-W only once, therefore no Bonferroni adjustment was needed. After significance was found we continued with post hoc analysis using Dwass-Steel-Critchlow-Fligner (DSCF) [15]. DSCF is a non-parametric post hoc test for pairwise comparison. It is generalized for unequal sample sizes [15] and has been shown to be more suitable for comparisons of non-normally distributions with unequal variances [13]. DSCF has built-in family-wise error rate protection [12, 15, 34], which automatically controls the error rate for all comparisons $[15,40]$, therefore p-value adjustment are no longer required when using DSCF. The statistical analysis was conducted using Pingouin and scikit-posthocs libraries in Python 3.8 [92, 99].

For subjective measurement of anxiety, we found a significant main effect of haptic patterns on participants' anxiety measured with STAI questionnaire $\left(\mathrm{H}(2)=14.39, p<0.001, \varepsilon^{2}=0.65\right)$. The post hoc test using DSCF revealed that participants experienced significantly lower anxiety, measured by STAI scores, with either thermal $($ Mean $=35.5, \mathrm{SD}=$ 
$12.5, p=0.003$ ) or vibrotactile patterns (Mean $=38.7, \mathrm{SD}=9.16, p=0.004)$, compared to those who received no haptic patterns $($ Mean $=56.8, \mathrm{SD}=3.11)$. While the number of participants is small and future work is needed to confirm it, an interesting outcome is that the STAI scores for each of these two haptic patterns suggest the potentially stronger beneficial impact of cool temperatures (Mean $=29.00, \mathrm{SD}=10.4$ ) and low frequency vibrations (less than or equal to 30 $\mathrm{bpm})($ Mean $=36.4, \mathrm{SD}=9.04)$ on subjective experience of anxiety, compared to warm temperatures $($ Mean $=42.00, \mathrm{SD}$ $=12.1)$ and high frequency vibrations (higher than $30 \mathrm{bpm})($ Mean $=44.5, \mathrm{SD}=9.19)$.

For objective measurement of stress, we found that the main effect of haptic patterns measured by RMSSD $(\mathrm{H}(2)=$ 5.40, $\left.p=0.067, \varepsilon^{2}=0.24\right)$ was approaching significance: the mean values of RMSSD under temperature (Mean $=32.4$, SD $=10.7)$ or vibration patterns $($ Mean $=48.2, \mathrm{SD}=37.6)$ were higher than the RMSSD under no haptic patterns $($ Mean $=$ 18.5, SD = 4.58), indicating again lower stress level for those participants experiencing haptic patterns. Differences in RMSSD values under warm-cool temperature patterns, and low-high frequency vibrotactile patterns were smaller on this objective measure of stress, compared to those identified on the subjective measures, albeit suggesting decreased levels of stress in the following order of patterns: cool temperatures, warm temperatures, high frequency vibrations, low frequency vibrations. These findings indicate differences in the objective and subjective measures of stress and anxiety assessed during the stressor task, and post stressor task, respectively. Nevertheless, low frequency vibrotactile patterns appear to be the most effective ones for stress regulation.

\subsection{Participants' Attitude towards Haptic Patterns for Affect Regulation in Everyday Life}

We now report on the main findings from the follow up interviews were we explored participants' attitude towards extending the use of their designed haptic patterns in daily life settings. When asked if they would consider using wrist-worn haptic technology for long term, participants envisioned situations where they could benefit from such devices. These included diverse activities in daily lives namely anxious events like exams, interviews or presentations, workplace and stressful meetings, when in fear or when worrying about future or confrontational situations. For Example, P1 (temperature) explained: "I want to use it for social anxiety. I would probably use it every day when I am in public. Every time when I get anxious". P2 (vibrations) added: "when you are at home, and you realize, you have got loads of things you have not done and you start to get stressed out about them. This [device] might be useful in giving me space to kind of step back and have a look at everything and calm down about the whole situation". P12 reported that warmth sensations in situations which are extremely stressful may not work: "I think this can be a useful tool to regulate oneself but in extreme [stress] situations, one might completely ignore it".

All participants appreciated haptic devices' privacy aspect: "I don't want it to show to others that I am using something to calm myself down" - P3. Similarly, P5 added: "I think the positive aspect is that these devices are not visual and they are hidden, so no one would know that you have a device that is trying to keep you calm". They also found wrist as suitable compared to other body locations for using it over long term: "I think the wrist is perfect [...] because it makes it wearable like a watch" - P7, however, they preferred such a technology to be integrated into existing smartwatches: "it is a hassle to have it only for this specific purpose, but it would be better if it can be incorporated in smartwatches which we can use for other purposes as well, so you can carry it around, just like a normal watch" - P13.

\section{Discussion}

We now revisit the initial research questions and reflect on the value of our findings for designing technologies for affect regulation. Our work has focused on exploring personalized vibrotactile and thermal patterns and their value in supporting affect regulation. 


\subsection{Distinct Experiential Qualities of Personalized Vibrotactile and Thermal Patterns}

5.1.1 Vibration Frequency and Rhythmic Entrainment. Findings indicate that participants perceived the vibrotactile patterns through three experiential qualities: heartbeat, rhythmic flow and slowness which have been limitedly explored in the context of affective health technologies [78]. These qualities, which are unpacked below, are partly rooted in the properties of the actuator whose range of vibration frequency is from 30 to $185 \mathrm{bpm}$, and intensity between 5 and 20 percent.

Heartbeat - Rhythmic flow: Entrainment: An important finding is that the participants' intuitive understanding of rhythm allowed them to associate vibration frequency to their heartbeat. This is important since participants could personalize the frequency of their vibration patterns from 30 to $185 \mathrm{bpm}$, which is a much broader range than that of adults' normal heartbeat, i.e., from 60 to $100 \mathrm{bpm}$ [2]. So although normal heartbeat is within the provided frequency range, the $30 \mathrm{bpm}$ chosen by most participants is a highly unlikely heartbeat, yet, surprisingly participants actually did associate it with heartbeat. Such insights echo consistent findings on rhythmic entrainment, a common phenomenon observed in physical and biological systems in which two rhythmic events interact with each other to eventually adapt and merge into a common phase [7]. In particular, the autonomic nervous system is characterized by rhythmic processes such as beating of our hearts or breathing. Because of the entrainment phenomenon, and these internal rhythms, people can synchronize them with external rhythms [17, 18, 42], irrespectively of these being at faster or slower pace than those of one's body. While playing with different vibration frequencies, participants intuitively experienced this dual aspect of entrainment, as high frequency vibrations made them anxious, confirming also previous findings on the impact of such vibrations on increased anxiety, and of slow vibrations on lower anxiety [10].

Heartbeat - Rhythmic flow - Slowness: Regulation through Entrainment: Our findings indicate that participants not only related vibration frequency to their heart rate, but to a heart rate in non anxious state, using the low pace of the vibration in order to down regulate their heart rate. Thus, participants valued the rhythmic flow of these slow vibrations which they could follow through entrainment, in order to slow themselves down during the stressor task. Consistent previous outcomes have also indicated the value of slowness for down regulation of affect, with many examples of sound in music therapy interventions [50], where slow tempo supported slower heart and respiration rate $[4,17]$, or tempo-based rhythm interventions at $60 \mathrm{bpm}$ promoted behavioral pacing, minimizing anxiety behaviors and facilitating concentration and calm [4, 9]. Slowness was a quality capturing both the slow tempo of the vibration, but also its lower intensity. Interestingly, slowness was appreciated even by participants who selected high intensity vibrations, as those choices were still motivated by down regulation, but from a higher heart rate baseline. Once, again, this reflects strong individual differences and the value of personalization of haptic patterns.

Heartbeat - Rhythmic flow - Slowness: Visceral: Another important aspect related to these qualities is the visceral experience of the vibrotactile patterns. Indeed, slow vibrations are visceral, felt almost within, allowing participants to follow their rhythm as if it was of their own heart. These findings support the view that with the right frequency, vibrations have the potential to be experienced as coming from the body, more specifically when linked to the rhythm of the cardiac system. In this light, we argue that the rhythmic entrainment facilitated by slow vibrations provides a strong embodiment to the intuitiveness, bodily-aliveness aspect of this vibrotactile pattern. In this way, such patterns may also start supporting awareness of one's heart rate, or interoceptive awareness [72] defined as awareness of one's own internal bodily signals. This is important, given that a wealth of findings have linked interoceptive awareness with emotional awareness and affect regulation [22, 71, 98], although such awareness takes time to develop. 
5.1.2 Warm and Cool-based Experiential Properties. Findings indicate that participants perceived thermal patterns through qualities such as subjectivity and focus; warm: touch and comfortable; cool: pleasant and noticeable; gradual changes, warm and cool: metaphors. These qualities which have been also limitedly explored in affective health technologies [78], are based on the actuators' ability to support warm and cool sensations within $+16^{\circ}$ to $-11^{\circ} \mathrm{C}$ range.

Thermal Patterns - Warm and Cool Sensations: Subjectivity and Focus: The overall impression from most participants was that the experience of thermal sensations tended to be subjective, and sensitive to the placement of actuator on different bodily locations. Participants felt that the experience of warm or cool temperature differs based on where they placed the actuator on the wrist. This is consistent with previous findings indicating that the thermal sensitivity differs across the body, with some areas being more sensitive than others where the same temperature is harder to detect [38]. This suggests the need for adjusting the temperature value according to the bodily location where it is experienced. Moreover, thermal patterns designed by participants attracted their attention during the stressor task. This is important, indicating the value of thermal patterns to distract attention from the stressor task and its negative high intensity affect.

Thermal Patterns - Warm Sensations: Touch and Comfortable: Reflecting on their felt body experiences, participants in our study described thermal experiences of warmth as comfortable and associated them to human touch. This is an important finding in the light of previous ones indicating that haptics can support affect regulation through affective touch which consists of gentle strokes through slow movement of warmth, and which has been shown to support secure attachment $[69,98]$. Recent work on the effects of tactile warmth has suggested that people associate the presence of warmth to social affiliation, and its absence to loneliness [19]. With respect to temperature, a wealth of studies have shown its intrinsic link with intimacy [105] and their impact on each other cued by either words or physical experience. In this respect, warmth relates to increased perception of intimacy [102], social proximity [35], social affiliation [19], trust [41] or warm personality [105]. A further illustration of this relationship between temperature and social affiliation is from psychotherapy research where client's warm hands have been associated with emotional security [60].

Thermal Patterns - Cool Sensations: Pleasant and Noticeable: Our study participants found the experience of cool temperature on their wrist to be unfamiliar yet pleasant. Moreover, those who chose cool temperatures indicated that the sensation of cool was more pleasant than the sensation of warm. This is important, given that previous work on the experiential qualities of temperature has looked mostly at heat, while cool temperature has been under-explored $[38,98]$. From the limited studies in this space, findings indicate that thermal stimuli consisting of cool temperature are more noticeable than warm temperature ones [106]. This is echoed by our findings highlighting the unfamiliar quality of cool temperature, thus its potential to better attract attention.

Thermal Patterns - Warm and Cool Sensations: Gradual: In contrast to vibrations, temperature changes are gradual. Actuators that provide thermal stimuli need to be turned off once they reach their target temperature, to allow for the dissipation of accumulated temperature, and be turned on again after the temperature has been dissipated [89, 98]. This pattern mitigates against temperature accumulation by alternating increases and decreases of actuated temperature towards the target temperature [65,98]. Interestingly, the thermal patterns explored in the study consisted of constant temperature, ensured by such gradual changes to avoid heat accumulation, albeit these changes were too subtle to be noticed. However, future work could explore dynamic thermal patterns where gradual changes can be noticed, contributing to a sense of rhythm. Indeed, previous findings on thermal sensations have confirmed that heat pulse consisting of gradual increase and decrease helped people to direct and sustain attention to different body parts [38, 89].

Thermal Patterns - Warm and Cool Sensations: Metaphors: Another important aspect of experiential qualities of thermal patterns is their metaphoric nature. Cognitive linguistics have explored the metaphors of emotions, some of 
which described with reference to temperature domain, i.e., "anger is heat", "he was cool to me" [47]. With respect to emotions, such work has highlighted the link between temperature and friendliness, with warmth being friendly and caring [101], which echos previous findings on the link between warmth and intimacy. Moreover, cool seemed to be an obvious choice for some participants as being angry or stressed is considered "feeling hot", and they wanted to "cool off" both physically and metaphorically. Research in cognitive linguistics have also identified temperature-based metaphors for emotional control or regulation such as hot is lack of emotional control, and cool is emotional control [101].

\subsection{Modalities of Haptic Patterns for Affect Regulation: Comparable Impact on Affect Regulation}

We now reflect on the value of users' designed haptic vibrotactile and thermal patterns for their affect regulation. Our study is one of the first to compare different haptic submodalities, contrasting most HCI work in this space, focusing predominantly on unimodal systems. We highlight the importance of such cross-modal comparison and their experiential qualities to better inform the design space of affect regulation technologies.

With respect to preferences, most participants (5 out of 7) designed vibrotactile patterns of low frequency, i.e., 30 $\mathrm{bpm}$. Preferences for thermal patterns were less consistent, with 4 out of 8 participants designing thermal patterns in the range of warm temperatures, i.e., between 2 and $10^{\circ} \mathrm{C}$, above the baseline, while the other 4 participants preferred cool temperatures, between -8 and $-11^{\circ} \mathrm{C}$, below the baseline.

Both subjective and objective measures indicate a lower level of anxiety and stress during the stressor task when participants experienced the haptic patterns compared to no haptic patterns. Descriptive statistics of the subjective measures of anxiety indicate lower anxiety under cool temperatures or low frequency vibrotactile patterns compared to warm temperatures and high frequency vibrations, respectively. In turn, descriptive statistics of objective measures of stress assessed during the stressor task indicate the lowest stress level under low frequency vibrotactile patterns, although no significant differences have emerged among the four patterns.

The value of cool temperature for lowering the subjective level of anxiety is particularly interesting. The fact that 4 out of 8 participants preferred cool temperatures is surprising, given their association with high rather than low stress. On the one hand, a wealth of findings indicate that changes in skin temperature reflect changes in the autonomous nervous system with an increase in peripheral blood flow and therefore in hands' and fingers' temperature during relaxation, and decrease of their temperature during stress [21, 66]. On the other hand, supported by everyday metaphors of cooling off, cool temperatures appear to offer a pleasant counterpart to the warmer body temperature experienced during stressful events [66]. Future work is needed to further explore the underpinning mechanisms of cool temperature patterns for affect regulation.

5.2.1 Designing Vibrotactile vs Thermal Patterns: Entrained to Slow Bodily Rhythms vs Expressive \& Hedonic. Although both vibrotactile and thermal patterns have comparable beneficial impact on affect regulation, our findings highlight that they ensure this in different ways. Indeed, participants' strong preferences for specific vibrotactile or thermal patterns, coupled with their rationale for such patterns, suggest that these two modalities may support affect regulation through different mechanisms. This is a key outcome that could broaden the focus of technologies for affect regulation from their current lens on if and how specific modality and actuation patterns work, to explore also why they work.

While vibrotactile patterns support entrainment to breathing or heart beating slow rhythms, thermal patterns are particularly hedonic and expressive, allowing for a broader range of choices. Indeed, participants' choices for personalizing haptic patterns is key, but such personalization in the case of vibrotactile patterns tends to be confined to the rather consistent biological rhythms of slow breathing or slow heart beating and thus particularly embodied. In 
contrast, thermal patterns can accommodate a larger range of both warmer and cooler personal preferences than the base temperature, characterized by expressive and hedonic qualities.

Our findings indicate the potentially stronger beneficial impact of low frequency vibrations on lowering stress level. This confirms the value of entrainment that systems based on slow heart rate patterns appear to support. These outcomes validate previous HCI work in this space that has prioritized haptic modalities and in particular low frequency vibrotactile patterns mapped to bodily rhythms such as slow heart rate. These have been illustrated by systems such as BoostMeUp [10], Doppel [3], or EmotionCheck [9], whose findings indicate that vibrotactile patterns with a frequency of $30 \%$ lower than the baseline heart rate support regulation of anxiety [10].

Although HCI work on affect regulation technologies is still in its early stages, in contrast to vibrotactile patterns, which have been more explored, there has been less focus on thermal patterns. Our findings confirm previous ones on the value of thermal feedback and particularly the expressiveness [98] of warm temperature for regulation [11] together with the hedonic qualities [89] of warm temperatures compared to cool ones. Our findings extend these prior works by showing the expressiveness and hedonic qualities of both warm and cool temperatures, while extend them with the exploration of a larger set of temperatures highlighting participants' preference for both warm and cool temperatures, albeit outside the hot and cold range.

5.2.2 Personalized Patterns for Affect Regulation. We now reflect on the choice of modalities, bodily rhythms, the dynamic adaption of designed patterns together with the value of such choices for affect regulation technologies.

Personalization: Choice of Modalities: Our outcomes confirm prior findings on the individual differences in sensitivity to temperature $[104,106]$ and vibrotactile stimuli $[6,100]$. More importantly, however, such individual differences have impacted participants' preferences for their designed haptic patterns, in terms of low-high vibration frequency and intensity, and warm-cool temperatures. In addition, despite the value of accounting for such preferences through personalization and IKEA effect [80], limited HCI work has involved users in the design of affective interfaces [84] and those that did, have focused mostly on visual modality [97]. In contrast, previous work on haptic patterns for affect regulation has paid limited attention to the personalization of both vibrotactile and thermal patterns.

With regard to vibrotactile patterns, unlike our work, systems such as BoostMeUp [10], Doppel [3] and EmotionCheck [9] that employed slow rhythmic vibrations linked to heart rate have not engaged users in their design. One exception here is Miri, and colleagues' recent work on their personalized breathing pacer, allowing users to set the frequency and amplitude of rhythmic vibrations to align with slow breathing for affect regulation [59]. In terms of thermal patterns, although there has been limited work exploring their value for regulation [89], the individual differences in users' sensitivity to heat and their appreciation of it have been acknowledged [38]. Such differences are rooted in skin sensitivity, personal experiences, and environmental factors [38, 75, 76], with the hedonic quality of thermal sensations being marked by internal body temperature [61]. Despite such individual differences in thermal perception, there has been surprisingly limited attention paid to users' personalization of thermal patterns.

This is an important gap that our work has started to address. Indeed, our outcomes highlight the importance of providing choices for personalization for both haptic modalities in terms of temperature intensity, as well as vibration frequency and intensity. Findings also indicate that participants appreciated such choices and the ability to vary important properties of each actuation, which led to specific experiential qualities for each haptic modality. Given people's preference for specific modalities reflected in different cognitive and perceptual styles [73], future work may explore users' preferences not only within the parameters of specific modalities, i.e., frequency and intensity of 
vibrotactile patterns, but also across haptic modalities, i.e., vibrotactile and thermal, or even across modalities, i.e., haptic and visual or aural already harnessed in entrainment technologies [79].

Personalization: Choice of Bodily Rhythms: Our findings indicate the value of vibrotactile patterns mapping slow heart rate to support what appears to be effortless synchronization of one's heart rate to such slow rhythm. These echo previous outcomes on vibrotactile patterns for affect regulation [3, 9, 10]. In contrast, research on affect regulation through slow breathing has just started to emerge more in the psychophysiology area [36, 88] with findings indicating its beneficial impact on the regulation [36]. In comparison to heart beating rhythm, breathing rhythm for affect regulation requires lower vibration frequency, i.e., 6 breaths/minute [88].

In HCI, research on slow breathing for affect regulation is rather limited; noticeable exceptions include BrightBeat [27] that provide visual and aural representations, rhythmically oscillating at slow speed, mapped to users' breathing rate in a relaxed state. Another example is the breathing pacer developed by Miri and colleagues [59] which has shown value for affect regulation. Miri and colleagues [59] introduced the distinction between explicit and implicit affect regulation and called for more research to compare their effectiveness. They defined implicit affect regulation as requiring merely perception or unconscious cognition and usually supported by systems involving slow heart rate rhythm, and explicit affect regulation requiring users' engagement at perception, cognition, and action level, usually supported by slow breathing-based systems. Miri and colleagues' findings indicate that explicit regulation of the slow breathing-based systems such as their breathing pacer is intentional and effortful, thus challenging the synchronization of one's breathing rhythm with that of the vibrotactile pattern, especially during stressor tasks.

Our findings indicate the value of entrainment that systems based on slow heart rate patterns appear to support. Most importantly, as reflected in our findings, such entrainment does not require effort. This contrasts the effortful synchronization of slow breathing supported by the breathing pacer system [59] while also echoing previous outcomes on the effortless quality of entrainment [27]. Arguably, unlike heart beating, breathing is an easier to perceive bodily function. Thus, when in one's focus of attention, it can be more difficult to regulate because real-time vibrotactile patterns, slower than one's current breathing rhythm, can counterproductively increase awareness of one's failure to regulate. Future work supporting effortless entrainment to slow breathing is much needed, especially since our findings indicate participants' preference for the lower frequency of the vibrotactile patterns, below $30 \mathrm{bpm}$. If such a choice can be provided, users could potentially choose to design vibrotactile patterns aligned not only with slow heart rate but even with slow breathing.

Adaptive Personalized Patterns: Another important outcome is that although the designed haptic patterns remained the same, participants mentioned that they would want to change the intensity of vibrotactile patterns or the absolute value of temperature ones according to how their stress varies throughout the stressor task. The reason for this is two-fold. First, constant patterns may lead to fatigue or habituation, thus limiting their impact. Indeed, the experience of a constant warm or cool temperature, or of a specific low or high frequency vibration on the same bodily placement, i.e., one's wrist over 10 minutes, tends to be tiring. Second, constant patterns are effective if the level of stress is constant too. However, even during the stressor task, the level of stress is likely to vary in time. Such variation of stress level and its demand for adaptive regulation patterns is insufficiently accommodated by constant patterns. To ensure adaptive patterns, biosensors measuring affect such as heart rate or galvanic skin response provided real-time data that can be used to inform the timing of actuation based on changes in stress level. Biosensors' data can also be used to adapt not just timing but also the intensity and duration of the actuation. In this way, the haptic patterns can be not just personalized by engaging users in their design, but also dynamically adapted to the real-time changes in stress level. 


\section{Implications for Designing Technologies for Affect Regulation}

This section contributes to the design of affect regulation technologies by providing implications for design entailed by our key findings. We highlight the i) value of supporting implicit affect regulation through entrainment of slow bodily rhythms, ii) decoupling such entrainment from the predominant vibrotactile modality, iii) designing for thermal biofeedback leveraging thermal patterns, and iv) supporting personalized and adaptive patterns.

\subsection{Design for Implicit Regulation: Entrainment of Slow Bodily Rhythms.}

HCI work on affective interfaces has explored both non-rhythmic and rhythmic vibrations. Non-rhythmic vibrations act more like notifications for the changes in physiological arousal, which per sei do not explicitly support affect regulation, albeit could prompt users to initiate it [98]. In contrast, rhythmic vibrotactile patterns at a frequency of the slow heart rate are associated with relaxation, therefore facilitate regulation through the synchronization of one's heart rate to such slow rhythm, $[3,9,10]$. Our outcomes support the value of entrainment to slow bodily rhythms, especially the effortless one to slow heart beating rhythm. In turn, such entrainment to bodily rhythms supports interoceptive awareness as the ability to recognize internal bodily states [11, 49] which has been shown to have strong wellbeing benefits, particularly for affect regulation [32, 57, 74]. However, in contrast to awareness of heartbeat, which requires minimal volitional control, awareness of breathing is effortful [23]. Thus, we encourage designers of affect regulation technologies interested in entrainment to focus on slow rhythmic patterns mapping heart beating rhythm and on future work to explore how to support effortless regulation of slow breathing.

\subsection{Entrainment of Slow Bodily Rhythms: Beyond Vibrotactile Modality.}

Although low frequency vibrations offer powerful and intuitive haptic modality to map slow bodily rhythms, we can further open up the design space of technologies for affect regulation. For instance, understanding the value of such patterns for supporting synchronization with slow bodily rhythms can lead to decoupling them from the current predominant haptic modality, namely vibrotactile patterns. Indeed, rhythm can be created through other sensory modalities such as visual or aural ones through dynamic stimuli that change rhythmically in time or even through a different haptic submodality in the form of thermal patterns designed to rhythmically change in time. The latter suggestion is particularly interesting as it could allow integrating the benefit of rhythmic entrainment, currently supported solely by vibrotactile patterns, with the expressive and hedonic qualities of thermal patterns. In other words, these insights open the design space of affect regulation technologies where modalities can be decoupled from the two key design principles that our findings have highlighted: entrainment to slow bodily rhythms; expressivity and hedonism.

\subsection{Design for Thermal Biofeedback.}

While low frequency vibrations offer effective haptic modality for affect regulation, thermal patterns were particularly valued for their expressiveness and hedonic qualities. As mentioned above, we suggest that they can be leveraged as rhythmic patterns designed for entrainment to slow bodily rhythm. Another way to harness thermal patterns is by drawing on the relationship between body temperature and stress: peripheral vasoconstriction leads to cool hands [21], and central vasodilation leads to a warm core or internal body temperatures [66, 67]. Thermal biofeedback is a therapeutic intervention for affect regulation involving training control of bodily functions in order to increase peripheral blood flow leading to higher skin temperature or warmed hands [70]. Prior findings have also shown that 
thermal biofeedback is facilitated if the temperature around the body is warmer [21, 104]. One can imagine thermal patterns of warm temperature that could support thermal biofeedback when integrated with its instructions. Such patterns, when applied to the wrist, could benefit from being integrated with wrist-worn photoplethysmography devices. One can also think of thermal patterns of cool temperatures utilized to lower core temperature, for instance, through placement on one's neck. The latter resonates well with our findings on participants' preference for cool thermal patterns, albeit their placement is more appropriate on central rather than peripheral bodily location.

\subsection{Support Personalized and Dynamically Adaptive Patterns.}

We want to emphasize the value of the user's personalizing their patterns for affect regulation. The choice for patterns' modality or bodily rhythm that can be entrained can increase users' expressiveness and their hedonic experience. We also suggest integrating the patterns' actuators with biosensors in order to provide real-time adaptive actuation. However, rather than being continuous, such actuation can be dynamic, varying with the changes in stress level. This move away from unchanged and continuous actuation mitigates against the risk of fatigue and habituation, increasing the potential for affect regulation.

\section{Conclusion}

This paper explored haptic modalities for affect regulation by engaging 23 participants in the creation of personalized vibrotactile and thermal patterns (high/low frequency and intensity, warm/cool). We report on the experiential qualities of these haptic patterns and their impact on affect regulation during stress elicitation tasks, for which we measured self-reported anxiety and heart rate variability. Findings indicate that subjective and objective measures of anxiety and stress were lower under haptic patterns than without, and that low frequency vibrations were the most effective patterns for stress regulation. The main contribution of this work includes a discussion of each haptic pattern's key qualities, i.e., vibrotactile frequency and rhythmic entrainment, warm and cool-based experiential properties. Our findings open up new design opportunities for regulation technologies for affective wellbeing. These include implicit affect regulation through entrainment of slow bodily rhythms beyond vibrotactile modality, design for thermal biofeedback patterns, and support for personalized and dynamically adaptive patterns.

\section{Acknowledgments}

This work has been supported by AffecTech: Personal Technologies for Affective Health, Innovative Training Network funded by the H2020 People Programme under Marie Skłodowska-Curie grant agreement No 722022.

\section{References}

[1] Miquel Alfaras, Vasiliki Tsaknaki, Pedro Sanches, Charles Windlin, Muhammad Umair, Corina Sas, and Kristina Höök. 2020. From Biodata to Somadata. In Proceedings of the 2020 CHI Conference on Human Factors in Computing Systems (Honolulu, HI, USA) (CHI '20). Association for Computing Machinery, New York, NY, USA, 1-14. https://doi.org/10.1145/3313831.3376684

[2] American Heart Association. 2015. All About Heart Rate (Pulse) | American Heart Association. https://www.heart.org/en/health-topics/highblood-pressure/the-facts-about-high-blood-pressure/all-about-heart-rate-pulse. (Accessed on 04/23/2021).

[3] Ruben T. Azevedo, Nell Bennett, Andreas Bilicki, Jack Hooper, Fotini Markopoulou, and Manos Tsakiris. 2017. The calming effect of a new wearable device during the anticipation of public speech. Scientific Reports 7, 1 (May 2017). https://doi.org/10.1038/s41598-017-02274-2

[4] Dorita S. Berger. 2012. Pilot Study Investigating the Efficacy of Tempo-Specific Rhythm Interventions in Music-Based Treatment Addressing Hyper-Arousal, Anxiety, System Pacing, and Redirection of Fight-or-Flight Fear Behaviors in Children with Autism Spectrum Disorder (ASD) Journal of Biomusical Engineering 2 (2012), 1-15. https://doi.org/10.4303/jbe/m110902

[5] Yekta Said Can, Heather Iles-Smith, Niaz Chalabianloo, Deniz Ekiz, Javier Fernández-Álvarez, Claudia Repetto, Giuseppe Riva, and Cem Ersoy. 2020. How to Relax in Stressful Situations: A Smart Stress Reduction System. In Healthcare, Vol. 8. Multidisciplinary Digital Publishing Institute, 100. 
[6] Roger W. Cholewiak and Amy A. Collins. 1997. Individual differences in the vibrotactile perception of a "simple" pattern set. Perception \& Psychophysics 59, 6 (Jan. 1997), 850-866. https://doi.org/10.3758/bf03205503

[7] Martin Clayton, Rebecca Sager, and Udo Will. 2005. In time with the music : the concept of entrainment and its significance for ethnomusicology. European meetings in ethnomusicology. 11 (ESEM Counterpoint 1) (January 2005), 1-82. http://dro.dur.ac.uk/8713/ With the permission of the publisher, the full-text of the complete issue of this journal has replaced the earlier author's manuscript version of this article.

[8] Desirée Colombo, Javier Fernández-Álvarez, Carlos Suso-Ribera, Pietro Cipresso, Hristo Valev, Tim Leufkens, Corina Sas, Azucena Garcia-Palacios, Giuseppe Riva, and Cristina Botella. 2020. The need for change: Understanding emotion regulation antecedents and consequences using ecological momentary assessment. Emotion 20, 1 (2020), 30.

[9] Jean Costa, Alexander T. Adams, Malte F. Jung, François Guimbretière, and Tanzeem Choudhury. 2016. EmotionCheck: Leveraging Bodily Signals and False Feedback to Regulate Our Emotions. In Proceedings of the 2016 ACM International foint Conference on Pervasive and Ubiquitous Computing (Heidelberg, Germany) (UbiComp '16). Association for Computing Machinery, New York, NY, USA, 758-769. https://doi.org/10.1145/2971648.2971752

[10] Jean Costa, François Guimbretière, Malte F. Jung, and Tanzeem Choudhury. 2019. BoostMeUp: Improving Cognitive Performance in the Moment by Unobtrusively Regulating Emotions with a Smartwatch. Proc. ACM Interact. Mob. Wearable Ubiquitous Technol. 3, 2, Article 40 (June 2019), 23 pages. https://doi.org/10.1145/3328911

[11] Claudia Daudén Roquet and Corina Sas. 2021. Interoceptive Interaction: An Embodied Metaphor Inspired Approach to Designing for Meditation. In Proceedings of the 2021 CHI Conference on Human Factors in Computing Systems (Yokohama, Japan) (CHI '21). Association for Computing Machinery, New York, NY, USA.

[12] Alex Dmitrienko, Christy Chuang-Stein, and Ralph B D’Agostino Sr. 2007. Pharmaceutical statistics using SAS: a practical guide. SAS Institute.

[13] Anil Dolgun and Haydar Demirhan. 2017. Performance of nonparametric multiple comparison tests under heteroscedasticity, dependency, and skewed error distribution. Communications in Statistics-Simulation and Computation 46, 7 (2017), 5166-5183.

[14] doppel. 2020. Feel calm and focused, naturally. https://feeldoppel.co.uk/. (Accessed on 07/28/2020).

[15] Critchlow E Douglas and Fligner A Michael. 1991. On distribution-free multiple comparisons in the one-way analysis of variance. Communications in Statistics-Theory and Methods 20, 1 (1991), 127-139.

[16] Jinlong Machinery \& Electronics. 2019. Doppel Transparent - See Through Thru Clear Case Coin Vibration Motor C1020B295. https://www.vibrationmotor.com/products/coin-vibration-motors/coin-vibrations-motors-with-brushes/c1020b295

[17] Robert J. Ellis, Julian Koenig, and Julian F. Thayer. 2012. Getting to the Heart: Autonomic Nervous System Function in the Context of Evidence-Based Music Therapy. Music and Medicine 4 (2012), 90-99.

[18] Joset A. Etzel, Erica L. Johnsen, Julie Dickerson, Daniel Tranel, and Ralph Adolphs. 2006. Cardiovascular and respiratory responses during musical mood induction. International fournal of Psychophysiology 61, 1 (July 2006), 57-69. https://doi.org/10.1016/j.ijpsycho.2005.10.025

[19] Adam J. Fay and Jon K. Maner. 2020. Interactive Effects of Tactile Warmth and Ambient Temperature on the Search for Social Affiliation. Social Psychology 51, 3 (May 2020), 199-204. https://doi.org/10.1027/1864-9335/a000407

[20] Jennifer Fereday and Eimear Muir-Cochrane. 2006. Demonstrating Rigor Using Thematic Analysis: A Hybrid Approach of Inductive and Deductive Coding and Theme Development. International Journal of Qualitative Methods 5, 1 (2006), 80-92. https://doi.org/10.1177/160940690600500107 arXiv:https://doi.org/10.1177/160940690600500107

[21] Matthew Fish, Carmen Russoniello, and Dominiquie M. Clemmons-James. 2018. The Use of Biofeedback in College Counseling. Fournal of College Counseling 21, 3 (Oct. 2018), 274-288. https://doi.org/10.1002/jocc.12109

[22] Jürgen Füstös, Klaus Gramann, Beate M. Herbert, and Olga Pollatos. 2012. On the embodiment of emotion regulation: interoceptive awareness facilitates reappraisal. Social Cognitive and Affective Neuroscience 8, 8 (Sept. 2012), 911-917. https://doi.org/10.1093/scan/nss089

[23] Sarah N. Garfinkel, Miranda F. Manassei, Giles Hamilton-Fletcher, Yvo In den Bosch, Hugo D. Critchley, and Miriam Engels. 2016. Interoceptive dimensions across cardiac and respiratory axes. Philosophical Transactions of the Royal Society B: Biological Sciences 371, 1708 (Nov. 2016 ), 20160014. https://doi.org/10.1098/rstb.2016.0014

[24] Tom Gayler, Corina Sas, and Vaiva Kalnikaite. 2020. Material Food Probe: Personalized 3D Printed Flavors for Emotional Communication in Intimate Relationships. In Proceedings of the 2020 ACM Designing Interactive Systems Conference (Eindhoven, Netherlands) (DIS '20). Association for Computing Machinery, New York, NY, USA, 965-978. https://doi.org/10.1145/3357236.3395533

[25] Tom Gayler, Corina Sas, and Vaiva Kalnikaite. 2021. Sensory Probes: An Exploratory Design Research Method for Human-Food Interaction. In Proceedings of the 2021 ACM Designing Interactive Systems Conference (DIS '21). Association for Computing Machinery, New York, NY, USA.

[26] Richard Gevirtz. 2013. The Promise of Heart Rate Variability Biofeedback: Evidence-Based Applications. Biofeedback 41, 3 (2013), 110-120.

[27] Asma Ghandeharioun and Rosalind Picard. 2017. BrightBeat: Effortlessly Influencing Breathing for Cultivating Calmness and Focus. In Proceedings of the 2017 CHI Conference Extended Abstracts on Human Factors in Computing Systems (Denver, Colorado, USA) (CHI EA '17). Association for Computing Machinery, New York, NY, USA, 1624-1631. https://doi.org/10.1145/3027063.3053164

[28] David Giles, Nick Draper, and William Neil. 2016. Validity of the Polar V800 heart rate monitor to measure RR intervals at rest. European journal of applied physiology 116, 3 (2016), 563-571.

[29] Anthony G Greenwald. 1976. Within-subjects designs: To use or not to use? Psychological Bulletin 83, 2 (1976), 314.

[30] James J. Gross. 1998. The Emerging Field of Emotion Regulation: An Integrative Review. Review of General Psychology 2, 3 (1998), $271-299$. https://doi.org/10.1037/1089-2680.2.3.271 arXiv:https://doi.org/10.1037/1089-2680.2.3.271 
[31] James J. Gross. 2015. Emotion Regulation: Current Status and Future Prospects. Psychological Inquiry 26, 1 (Jan. 2015), 1-26. https://doi.org/10. 1080/1047840x.2014.940781

[32] Adam W. Hanley, Wolf E. Mehling, and Eric L. Garland. 2017. Holding the body in mind: Interoceptive awareness, dispositional mindfulness and psychological well-being. Journal of Psychosomatic Research 99 (Aug. 2017), 13-20. https://doi.org/10.1016/j.jpsychores.2017.05.014

[33] Dimitra Hartas. 2015. Educational research and inquiry: Qualitative and quantitative approaches. Bloomsbury Publishing.

[34] Myles Hollander, Douglas A Wolfe, and Eric Chicken. 2013. Nonparametric statistical methods. Vol. 751. John Wiley \& Sons.

[35] Hans IJzerman and Gün R. Semin. 2009. The Thermometer of Social Relations. Psychological Science 20, 10 (Oct. 2009), 1214-1220. https: //doi.org/10.1111/j.1467-9280.2009.02434.x

[36] Ravinder Jerath, Molly W. Crawford, Vernon A. Barnes, and Kyler Harden. 2015. Self-Regulation of Breathing as a Primary Treatment for Anxiety. Applied Psychophysiology and Biofeedback 40, 2 (April 2015), 107-115. https://doi.org/10.1007/s10484-015-9279-8

[37] Lynette Jones. 2009. Thermal touch. Scholarpedia 4, 5 (2009), 7955. https://doi.org/10.4249/scholarpedia.7955

[38] Martin Jonsson, Anna Ståhl, Johanna Mercurio, Anna Karlsson, Naveen Ramani, and Kristina Höök. 2016. The Aesthetics of Heat: Guiding Awareness with Thermal Stimuli. In Proceedings of the TEI '16: Tenth International Conference on Tangible, Embedded, and Embodied Interaction (Eindhoven, Netherlands) (TEI '16). Association for Computing Machinery, New York, NY, USA, 109-117. https://doi.org/10.1145/2839462.2839487 [39] Laura J Julian. 2011. Measures of anxiety. Arthritis care \& research 63, 011 (2011).

[40] Paul Juneau. 2004. Simultaneous nonparametric inference in a one-way layout using the SAS System. In Proceedings of the PharamSUG 2004 Annual Meeting.

[41] Yoona Kang, Lawrence E. Williams, Margaret S. Clark, Jeremy R. Gray, and John A. Bargh. 2010. Physical temperature effects on trust behavior: the role of insula. Social Cognitive and Affective Neuroscience 6, 4 (Aug. 2010), 507-515. https://doi.org/10.1093/scan/nsq077

[42] Stéphanie Khalfa, Mathieu Roy, Pierre Rainville, Simone Dalla Bella, and Isabelle Peretz. 2008. Role of tempo entrainment in psychophysiological differentiation of happy and sad music? International fournal of Psychophysiology 68, 1 (April 2008), 17-26. https://doi.org/10.1016/j.ijpsycho.2007. 12.001

[43] Hye Geum Kim, Eun Jin Cheon, Dai Seg Bai, Young Hwan Lee, and Bon Hoon Koo. 2018. Stress and heart rate variability: A meta-analysis and review of the literature. Psychiatry Investigation 15, 3 (mar 2018), 235-245. /pmc/articles/PMC5900369/?report=abstracthttps:/www.ncbi.nlm.nih. gov/pmc/articles/PMC5900369/

[44] Clemens Kirschbaum, Karl-Martin Pirke, and Dirk H Hellhammer. 1993. The 'Trier Social Stress Test'-a tool for investigating psychobiological stress responses in a laboratory setting. Neuropsychobiology 28, 1-2 (1993), 76-81.

[45] Sylvain Laborde, Emma Mosley, and Julian F Thayer. 2017. Heart rate variability and cardiac vagal tone in psychophysiological researchrecommendations for experiment planning, data analysis, and data reporting. Frontiers in psychology 8 (2017), 213.

[46] Embr - Embr Labs. 2020. Own Your Temperature | Feel Colder or Warmer. https://embrlabs.com/. (Accessed on 07/28/2020).

[47] George Lakoff and Zoltán Kövecses. 1987. The cognitive model of anger inherent in American English. In Cultural Models in Language and Thought. Cambridge University Press, 195-221. https://doi.org/10.1017/cbo9780511607660.009

[48] Pollyana Caldeira Leal, Tiago Costa Goes, Luiz Carlos Ferreira da Silva, and Flavia Teixeira-Silva. 2017. Trait vs. state anxiety in different threatening situations. Trends in psychiatry and psychotherapy 39, 3 (2017), 147-157.

[49] Adam Lobel, Marientina Gotsis, Erin Reynolds, Michael Annetta, Rutger C.M.E. Engels, and Isabela Granic. 2016. Designing and Utilizing Biofeedback Games for Emotion Regulation: The Case of Nevermind. In Proceedings of the 2016 CHI Conference Extended Abstracts on Human Factors in Computing Systems (San Jose, California, USA) (CHI EA '16). Association for Computing Machinery, New York, NY, USA, $1945-1951$. https://doi.org/10.1145/2851581.2892521

[50] Joanne Loewy. 2015. NICU music therapy: song of kin as critical lullaby in research and practice. Annals of the New York Academy of Sciences 1337 , 1 (March 2015), 178-185. https://doi.org/10.1111/nyas.12648

[51] Diana MacLean, Asta Roseway, and Mary Czerwinski. 2013. MoodWings: A Wearable Biofeedback Device for Real-Time Stress Intervention. In Proceedings of the 6th International Conference on PErvasive Technologies Related to Assistive Environments (Rhodes, Greece) (PETRA '13). Association for Computing Machinery, New York, NY, USA, Article 66, 8 pages. https://doi.org/10.1145/2504335.2504406

[52] Mark E. Maruish. 1994. Use of psychological testing for treatment planning and outcome assessment. Lawrence Erlbaum.

[53] Margaret W Matlin and Hugh J Foley. 1992. Sensation and perception. Allyn \& Bacon.

[54] Rollin McCraty and Fred Shaffer. 2015. Heart Rate Variability: New Perspectives on Physiological Mechanisms, Assessment of Self-regulatory Capacity, and Health risk. Global advances in health and medicine 4, 1 (jan 2015), 46-61. http://www.ncbi.nlm.nih.gov/pubmed/25694852http: //www.pubmedcentral.nih.gov/articlerender.fcgi?artid=PMC4311559

[55] Daniel McDuff, Amy Karlson, Ashish Kapoor, Asta Roseway, and Mary Czerwinski. 2012. AffectAura: An Intelligent System for Emotional Memory. In Proceedings of the SIGCHI Conference on Human Factors in Computing Systems (Austin, Texas, USA) (CHI '12). Association for Computing Machinery, New York, NY, USA, 849-858. https://doi.org/10.1145/2207676.2208525

[56] John David McElrath. 2015. The Effects of Pressure on Executive Functioning Skills during Computer-Based and Functional Performance Testing. Ph.D. Dissertation. Stephen F. Austin State University.

[57] Wolf Mehling. 2016. Differentiating attention styles and regulatory aspects of self-reported interoceptive sensibility. Philosophical Transactions of the Royal Society B: Biological Sciences 371, 1708 (Nov. 2016), 20160013. https://doi.org/10.1098/rstb.2016.0013 
[58] Pardis Miri, Robert Flory, Andero Uusberg, Helen Uusberg, James J. Gross, and Katherine Isbister. 2017. HapLand: A Scalable Robust Emotion Regulation Haptic System Testbed. In Proceedings of the 2017 CHI Conference Extended Abstracts on Human Factors in Computing Systems (Denver, Colorado, USA) (CHI EA '17). Association for Computing Machinery, New York, NY, USA, 1916-1923. https://doi.org/10.1145/3027063.3053147

[59] Pardis Miri, Emily Jusuf, Andero Uusberg, Horia Margarit, Robert Flory, Katherine Isbister, Keith Marzullo, and James J. Gross. 2020. Evaluating a Personalizable, Inconspicuous Vibrotactile(PIV) Breathing Pacer for In-the-Moment Affect Regulation. In Proceedings of the 2020 CHI Conference on Human Factors in Computing Systems (Honolulu, HI, USA) (CHI '20). Association for Computing Machinery, New York, NY, USA, 1-12. https://doi.org/10.1145/3313831.3376757

[60] Bela Mittelmann and Harold G. Wolff. 1943. Emotions and Skin temperature: Observations on Patients During Psychotherapeutic (Psychoanalytic) Interviews1. Psychosomatic Medicine 5, 3 (July 1943), 211. https://doi.org/10.1097/00006842-194307000-00002

[61] George D. Mower. 1976. Perceived intensity of peripheral thermal stimuli is independent of internal body temperature. fournal of Comparative and Physiological Psychology 90, 12 (1976), 1152-1155. https://doi.org/10.1037/h0077284

[62] Markus Neuhäuser and Frank Bretz. 2001. Nonparametric all-pairs multiple comparisons. Biometrical fournal: fournal of Mathematical Methods in Biosciences 43, 5 (2001), 571-580.

[63] Susan Nolen-Hoeksema. 2019. Abnormal psychology. McGraw-Hill Education.

[64] Jan M Noyes and Daniel PJ Bruneau. 2007. A self-analysis of the NASA-TLX workload measure. Ergonomics 50, 4 (2007), $514-519$.

[65] William Odom, Richard Banks, Abigail Durrant, David Kirk, and James Pierce. 2012. Slow Technology: Critical Reflection and Future Directions. In Proceedings of the Designing Interactive Systems Conference (Newcastle Upon Tyne, United Kingdom) (DIS '12). Association for Computing Machinery, New York, NY, USA, 816-817. https://doi.org/10.1145/2317956.2318088

[66] Jaka Ogorevc, Anja Podlesek, Gregor Gersak, and Janko Drnovsek. 2011. The effect of mental stress on psychophysiological parameters. In 2011 IEEE International Symposium on Medical Measurements and Applications. IEEE. https://doi.org/10.1109/memea.2011.5966692

[67] Takakazu Oka, Kae Oka, and Tetsuro Hori. 2001. Mechanisms and Mediators of Psychological Stress-Induced Rise in Core Temperature. Psychosomatic Medicine 63, 3 (May 2001), 476-486. https://doi.org/10.1097/00006842-200105000-00018

[68] Pablo E. Paredes, Yijun Zhou, Nur Al-Huda Hamdan, Stephanie Balters, Elizabeth Murnane, Wendy Ju, and James A. Landay. 2018. Just Breathe: In-Car Interventions for Guided Slow Breathing. Proc. ACM Interact. Mob. Wearable Ubiquitous Technol. 2, 1, Article 28 (March 2018 ), 23 pages. https://doi.org/10.1145/3191760

[69] Rebecca Anne Parker. 2012. The effects of physical touch and thermal warmth on interpersonal trust. (2012).

[70] CJ Peek. 2016. A primer of traditional biofeedback instrumentation. Biofeedback: A practitioner's guide (2016), 35-67.

[71] Olga Pollatos, Wladimir Kirsch, and Rainer Schandry. 2005. On the relationship between interoceptive awareness, emotional experience, and brain processes. Cognitive Brain Research 25, 3 (Dec. 2005), 948-962. https://doi.org/10.1016/j.cogbrainres.2005.09.019

[72] Olga Pollatos, Eva Traut-Mattausch, Heike Schroeder, and Rainer Schandry. 2007. Interoceptive awareness mediates the relationship between anxiety and the intensity of unpleasant feelings. Fournal of Anxiety Disorders 21, 7 (Jan. 2007), 931-943. https://doi.org/10.1016/j.janxdis.2006.12.004

[73] Stephen Rayner and Richard Riding. 1997. Towards a Categorisation of Cognitive Styles and Learning Styles. Educational Psychology 17, 1-2 (March 1997), 5-27. https://doi.org/10.1080/0144341970170101

[74] J. Brendan Ritchie and Peter Carruthers. 2013. The Bodily Senses. Oxford University Press. https://doi.org/10.1093/oxfordhb/9780199600472.013.026

[75] Katri Salminen, Veikko Surakka, Jani Lylykangas, Jukka Raisamo, Rami Saarinen, Roope Raisamo, Jussi Rantala, and Grigori Evreinov. 2008. Emotional and Behavioral Responses to Haptic Stimulation. In Proceedings of the SIGCHI Conference on Human Factors in Computing Systems (Florence, Italy) (CHI '08). Association for Computing Machinery, New York, NY, USA, 1555-1562. https://doi.org/10.1145/1357054.1357298

[76] Katri Salminen, Veikko Surakka, Jukka Raisamo, Jani Lylykangas, Roope Raisamo, Kalle Mäkelä, and Teemu Ahmaniemi. 2013. Cold or Hot? How Thermal Stimuli Are Related to Human Emotional System? In Haptic and Audio Interaction Design. Springer Berlin Heidelberg, 20-29. https://doi.org/10.1007/978-3-642-41068-0_3

[77] Pedro Sanches, Kristina Höök, Corina Sas, and Anna Ståhl. 2019. Ambiguity as a Resource to Inform Proto-Practices: The Case of Skin Conductance. ACM Trans. Comput.-Hum. Interact. 26, 4, Article 21 (July 2019), 32 pages. https://doi.org/10.1145/3318143

[78] Pedro Sanches, Axel Janson, Pavel Karpashevich, Camille Nadal, Chengcheng Qu, Claudia Daudén Roquet, Muhammad Umair, Charles Windlin, Gavin Doherty, Kristina Höök, and Corina Sas. 2019. HCI and Affective Health: Taking Stock of a Decade of Studies and Charting Future Research Directions. In Proceedings of the 2019 CHI Conference on Human Factors in Computing Systems (Glasgow, Scotland Uk) (CHI '19). Association for Computing Machinery, New York, NY, USA, 1-17. https://doi.org/10.1145/3290605.3300475

[79] Corina Sas and Rohit Chopra. 2015. MeditAid: a wearable adaptive neurofeedback-based system for training mindfulness state. Personal and Ubiquitous Computing 19, 7 (Sept. 2015), 1169-1182. https://doi.org/10.1007/s00779-015-0870-z

[80] Corina Sas and Carman Neustaedter. 2017. Exploring DIY Practices of Complex Home Technologies. ACM Trans. Comput.-Hum. Interact. 24, 2, Article 16 (April 2017), 29 pages. https://doi.org/10.1145/3057863

[81] Federica Scarpina and Sofia Tagini. 2017. The stroop color and word test. Frontiers in Psychology 8 (apr 2017). https://doi.org/10.3389/fpsyg.2017.00557

[82] Hasti Seifi and Karon E. MacLean. 2017. Exploiting haptic facets: Users' sensemaking schemas as a path to design and personalization of experience. International fournal of Human-Computer Studies 107 (Nov. 2017), 38-61. https://doi.org/10.1016/j.ijhcs.2017.04.003

[83] Fred Shaffer and J P Ginsberg. 2017. An Overview of Heart Rate Variability Metrics and Norms. Frontiers in Public Health 5 (2017), 258. http://www.ncbi.nlm.nih.gov/pubmed/29034226http://www.pubmedcentral.nih.gov/articlerender.fcgi?artid=PMC5624990 
[84] Will Simm, Maria Angela Ferrario, Adrian Gradinar, Marcia Tavares Smith, Stephen Forshaw, Ian Smith, and Jon Whittle. 2016. Anxiety and Autism: Towards Personalized Digital Health. In Proceedings of the 2016 CHI Conference on Human Factors in Computing Systems (San Jose, California, USA) (CHI '16). Association for Computing Machinery, New York, NY, USA, 1270-1281. https://doi.org/10.1145/2858036.2858259

[85] Matthew J. Smith, Kristen Warren, David Cohen-Tanugi, Sam Shames, Kelly Sprehn, Jana L. Schwartz, Hui Zhang, and Ed Arens. 2017. Augmenting Smart Buildings and Autonomous Vehicles with Wearable Thermal Technology. In Human-Computer Interaction. Interaction Contexts, Masaaki Kurosu (Ed.). Springer International Publishing, Cham, 550-561.

[86] Charles D Spielberger, Richard L Gorsuch, R Lushene, Peter R Vagg, and Gerard A Jacobs. 1983. Manual for the state-trait anxiety inventory (Palo Alto, CA, Consulting Psychologists Press). Inc (1983).

[87] Anna Ståhl, Kristina Höök, Martin Svensson, Alex S. Taylor, and Marco Combetto. 2008. Experiencing the Affective Diary. Personal and Ubiquitous Computing 13, 5 (June 2008), 365-378. https://doi.org/10.1007/s00779-008-0202-7

[88] Patrick R. Steffen, Tara Austin, Andrea DeBarros, and Tracy Brown. 2017. The Impact of Resonance Frequency Breathing on Measures of Heart Rate Variability, Blood Pressure, and Mood. Frontiers in Public Health 5 (Aug. 2017). https://doi.org/10.3389/fpubh.2017.00222

[89] Anna Ståhl, Martin Jonsson, Johanna Mercurio, Anna Karlsson, Kristina Höök, and Eva-Carin Banka Johnson. 2016. The Soma Mat and Breathing Light. In Proceedings of the 2016 CHI Conference Extended Abstracts on Human Factors in Computing Systems (San Jose, California, USA) (CHI EA '16). Association for Computing Machinery, New York, NY, USA, 305-308. https://doi.org/10.1145/2851581.2889464

[90] J Ridley Stroop. 1935. Studies of interference in serial verbal reactions. Journal of experimental psychology 18, 6 (1935), 643.

[91] Joachim Taelman, Steven Vandeput, Arthur Spaepen, and Sabine Van Huffel. 2009. Influence of mental stress on heart rate and heart rate variability. In 4th European conference of the international federation for medical and biological engineering. Springer, 1366-1369.

[92] Maksim A Terpilowski. 2019. scikit-posthocs: Pairwise multiple comparison tests in Python. fournal of Open Source Software 4, 36 (2019), 1169.

[93] Masahiko Tsukuda, Yukari Nishiyama, Shikiho Kawai, and Yasuaki Okumura. 2019. Identifying stress markers in skin gases by analysing gas collected from subjects undergoing the Trier social stress test and performing statistical analysis. fournal of breath research 13, 3 (2019), 036003.

[94] Muhammad Umair, Niaz Chalabianloo, Corina Sas, and Cem Ersoy. 2021. HRV and Stress: A Mixed-Methods Approach for Comparison of Wearable Heart Rate Sensors for Biofeedback. IEEE Access 9 (2021), 14005-14024. https://doi.org/10.1109/access.2021.3052131

[95] Muhammad Umair, Muhammad Hamza Latif, and Corina Sas. 2018. Dynamic Displays at Wrist for Real Time Visualization of Affective Data. In Proceedings of the 2018 ACM Conference Companion Publication on Designing Interactive Systems (Hong Kong, China) (DIS '18 Companion). Association for Computing Machinery, New York, NY, USA, 201-205. https://doi.org/10.1145/3197391.3205436

[96] Muhammad Umair and Corina Sas. 2019. Problems in Practice: Critiquing on Thermochromic based Displays for Representing Skin Conductance. (2019), 5 pages. https://eprints.lancs.ac.uk/id/eprint/131738/4/Muhammad_Umair_Critiquing_on_Thermochromic_based_Displays_for_ Representing_Skin_Conductance.pdf

[97] Muhammad Umair, Corina Sas, and Miquel Alfaras. 2020. ThermoPixels: Toolkit for Personalizing Arousal-Based Interfaces through Hybrid Crafting. In Proceedings of the 2020 ACM Designing Interactive Systems Conference (Eindhoven, Netherlands) (DIS '20). Association for Computing Machinery, New York, NY, USA, 1017-1032. https://doi.org/10.1145/3357236.3395512

[98] Muhammad Umair, Corina Sas, and Muhammad Hamza Latif. 2019. Towards Affective Chronometry: Exploring Smart Materials and Actuators for Real-Time Representations of Changes in Arousal. In Proceedings of the 2019 on Designing Interactive Systems Conference (San Diego, CA, USA) (DIS '19). Association for Computing Machinery, New York, NY, USA, 1479-1494. https://doi.org/10.1145/3322276.3322367

[99] Raphael Vallat. 2018. Pingouin: statistics in Python. Journal of Open Source Software 3, 31 (2018), 1026.

[100] Jan BF Van Erp et al. 2002. Guidelines for the use of vibro-tactile displays in human computer interaction. In Proceedings of eurohaptics, Vol. 2002. Citeseer, $18-22$.

[101] Susanne Vejdemo and Sigi Vandewinkel. 2016. 9. Extended uses of body-related temperature expressions. In The Lexical Typology of Semantic Shifts. De Gruyter. https://doi.org/10.1515/9783110377675-009

[102] Matthew Vess. 2012. Warm Thoughts. Psychological Science 23, 5 (April 2012), 472-474. https://doi.org/10.1177/0956797611435919

[103] Bernadette Von Dawans, Clemens Kirschbaum, and Markus Heinrichs. 2011. The Trier Social Stress Test for Groups (TSST-G): A new research tool for controlled simultaneous social stress exposure in a group format. Psychoneuroendocrinology 36, 4 (2011), 514-522.

[104] Zhe Wang, Richard de Dear, Maohui Luo, Borong Lin, Yingdong He, Ali Ghahramani, and Yingxin Zhu. 2018. Individual difference in thermal comfort: A literature review. Building and Environment 138 (June 2018), 181-193. https://doi.org/10.1016/j.buildenv.2018.04.040

[105] Lawrence E. Williams and John A. Bargh. 2008. Experiencing Physical Warmth Promotes Interpersonal Warmth. Science 322, 5901 (Oct. 2008), 606-607. https://doi.org/10.1126/science.1162548

[106] Graham Wilson, Martin Halvey, Stephen A. Brewster, and Stephen A. Hughes. 2011. Some like It Hot: Thermal Feedback for Mobile Devices. In Proceedings of the SIGCHI Conference on Human Factors in Computing Systems (Vancouver, BC, Canada) (CHI '11). Association for Computing Machinery, New York, NY, USA, 2555-2564. https://doi.org/10.1145/1978942.1979316

[107] Bin Yu, Jun Hu, Mathias Funk, and Loe M. G. Feijs. 2018. DeLight: biofeedback through ambient light for stress intervention and relaxation assistance. Personal and Ubiquitous Computing 22 (2018), 787-805. 